\title{
Determinación de la asociación del polimorfismo de la Apolipoproteína E (Apo E) relacionada con la presencia de placa aterosclerótica coronaria.
}

\author{
Adriana Moreno Valladares Bsc ${ }^{1}$, Ana Lucia Torres PhD', Álvaro E. Cartagena P MD ${ }^{2,3}$, Maria Helena Herrera MD. \\ ${ }^{1}$ Grupo de investigación: Clínico - Genético - Molecular en Dislipoproteínemias Pontificia Universidad Javeriana. Línea Biomarcadores \\ en Enfermedad Cardiovascular \\ ${ }^{2}$ Grupo de Medicina Interna y Cardiología. Hospital San Blas. \\ ${ }^{3}$ Docente cardiología Universidad Nacional de Colombia. Facultad de Medicina. \\ ${ }^{4}$ Grupo de Medicina Interna y Cardiología. Hospital Santa Clara. \\ Correspondencia: amorenov13@unicolmayor.edu.co.
}

Recibido: 08-03-2006 / Aceptado:03-07-2006

\begin{abstract}
Resumen
El polimorfismo de la Apolipoproteína E (Apo E) juega un papel clave en el metabolismo de lípidos y la enfermedad coronaria. Este estudio buscó identificar polimorfismos específicos de Apo E por PCR-RFLP y determinar la asociación de estas formas alelicas con la presencia de placa ateroesclerótica. Se analizó un total de 122 pacientes entre 29 y 85 años de edad con angina o infarto agudo del miocardio, en donde la presencia de oclusión se determinó por arteriografía coronaria. Del total de pacientes analizados, solo el 48.4\% presentaban un grado de obstrucción mayor del 70\%. De estos pacientes 40 fueron hombres y 19 mujeres. Al establecer la asociación entre grados de obstrucción y polimorfismo de ApoE estratificada por genero, se obtuvo un $\mathrm{p}=0.0054$ para los hombres y un $\mathrm{p}=0.977$ para las mujeres, lo que indica que esta asociación es significativa únicamente para el genero masculino. El genotipo E2/2 se asocio con no presencia de obstrucción. En conclusión este estudio permitió identificar el alelo E3/3 como altamente significante en la obstrucción coronaria dependiente del sexo así como confirma lo planteado por muchos autores a nivel internacional y nacional, que el alelo E2 pudiera tener un efecto antiaterogenico importante, al igual que la isoforma E4/4 no se encuentra relacionada con obstrucción coronaria.

El efecto a nivel cardiovascular de la ApoE sigue sin ser entendido es por esto que amerita mas estudios y evidenciar un comportamiento mas certero, lo que supone futuras investigaciones.

Palabras claves: Apolipoproteina E, dislipidemia, polimorfismo, genotipo, ateroesclerosis.

Abstract

The polimorfism of the Apolipoproteín E (Apo E), it plays a key paper in the lípids metabolism and the coronary illness. This study looked for to identify specific polimorfisms of Apo E for PCR-RFLP and to determine the association in these ways alelicas with the presence of plaque atheroesclerotic. A total of 122 patients was analyzed between 29 and 85 years old, with angina or sharp infart of the miocardial where the occlusion presence was determined by coronary arteriographic. Of the total of analyzed patients, alone $48.4 \%$ presented an obstruction degree bigger than $70 \%$. Of these patients 40 were men and 19 women. When establishing the association among obstruction degrees and polimorfismo of ApoE stratified for the genders, a
\end{abstract}


"p" was obtained $=0.0054$ for the men and a " $p "=0.977$ for the women, what indicates that this association is significant only for the genders masculine. The genotipo E2/2 you associates with they doesn't witness of obstruction.In conclusion, this study allowed to identifications of allelo E3/3 highly significant in coronary obstruction dependent on gender, confirming what is stated by many authors at national and international level in the sense that genotype E2, could have an important antiatherogenic effect, the same as the isoform E4/4 is not related with coronary obstruction.

The effect of the ApoE at cardiovascular level has not been completely clarified yet, what makes it whorty for more studies to demonstrate a more accurate behavior.

Key words: Apolipoprotein E, plaque atheroesclerotic, polimorfism, genotipo.

\section{Introducción}

Dada la alta tasa de prevalencia de la enfermedad coronaria a nivel nacional y mundial, es de suma importancia definir claramente la implicación de algunos factores que hasta el momento no han sido muy esclarecidos $(19.8 \%)$ y pudieran tener un impacto importante en su génesis (1).

Aunque es bien conocido que el origen de la enfermedad coronaria es multifactorial y poligénico, muchos de estos factores o agentes que participan en la cadena de la enfermedad no se han establecido con precisión, de hecho es el caso de moléculas emergentes importantes implicadas en el metabolismo del colesterol y triglicéridos como lo son las Apolipoproteínas y específicamente la Apolipoproteína E (ApoE) (2).

La ApoE se ubica en el cromosoma 19 locus q13.2q13.3 mas precisamente entre los pares de bases $50,100,901$ a 50,104,488, con un tamaño de 3597 pb, consta de 4 exones y tres intrones (3). Es una glicoproteína polimórfica cuyos alelos originan 3 genotipos homocigotos: E2/2, E3/3, E4/4, y tres heterocigotos: E3/2, E4/2, E4/3 (4). Dichas isoformas se diferencian entre sí por sustituciones de dos aminoácidos en los codones 112 y 158 así: la isoforma Apo E3 presenta Cisteína en la posición 112 y Arginina en la posición 158, en la Apo E2 se sustituye por Cisteína la posición 158 y en Apo E4 la Arginina ocupa la posición 112, teniendo en cuenta estas sustituciones la Apo E3 se considera la forma salvaje $(5,6)$.

Se ha establecido que, la Apo E se sintetiza en el hígado y está presente en las lipoproteínas, VLDL,
IDL, HDL y los remanentes de quilomicrones (7). Regula la extracción de los restos de lipoproteínas del plasma, por parte del hígado (8). No hay duda que esta lipoproteína juega un papel central en el metabolismo del colesterol y los triglicéridos (TG), ya que participa en el transporte de lípidos desde el hígado a las células periféricas: de hecho es una molécula estructural esencial de quilomicrones, VLDL, sus partículas remantes y HDL. (9).

En estudios anteriores se demostró que el sitio de unión a uno de los receptores (LDL-R) se encuentra en la porción amino terminal entre los aminoácidos 141 y 155 de la Apo E, Figura 1, que cumple función antioxidante (10). Cuando las lipoproteínas se unen al receptor comienza la captación y degradación de estas por parte de la célula, esto permite la utilización del colesterol contenido en las lipoproteínas, dándose la regulación intracelular de este colesterol (11).

En general, esta variación genética la convierte en una molécula de gran interés e impacto a nivel cardiovascular ya que juega un papel esencial en la unión a receptores de remanentes de quilomicrones, VLDL así como de LDL $(12,13)$.

Numerosos estudios han demostrado que las LDL son las lipoproteína con mayor y mas evidente poder aterogénico; de hecho, cualquier nivel de colesterol mayor de $100 \mathrm{mg} / \mathrm{dl}$ parece favorecer la aterogénesis, estas son mas susceptibles a la oxidación y a producir daño arterial. Algunas proteínas ricas en triglicéridos también han evidenciado ser aterogénicas, fundamentalmente los remanentes de VLDL o IDL ya que po- 


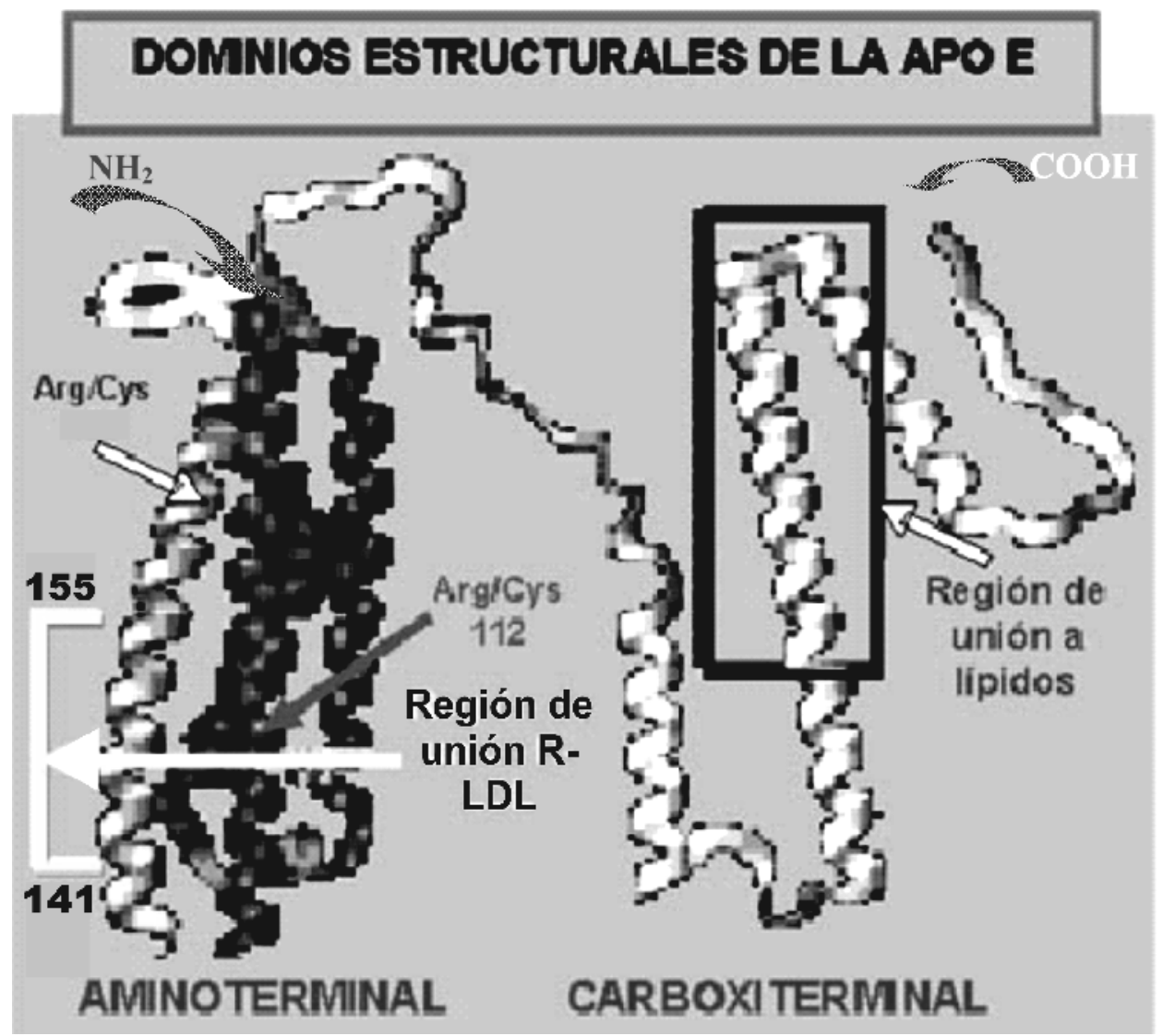

Figura 1. Estructura de la Apolipoproteína E., de 299 amino ácidos con un peso molecular de 34200 Daltons .

seen muchas de las propiedades de las LDL. La suma del colesterol transportado por las VLDL y las LDL constituye el "colesterol no HDL". Es bien conocido que el efecto de niveles altos de colesterol en el plasma, especialmente los que se asocian a lipoproteínas de baja densidad o LDL, aumentan el riesgo de desarrollar ateroesclerosis, por lo tanto, concentraciones elevadas de LDL se asocian con deterioro de la función endotelial de la intima arterial (14).

De hecho el estudio de un polimorfismo, puede llegar a ser una herramienta diagnostica o predictiva de gran ayuda para enfermedades que pudieran desarrollarse más adelante o identificar su predisposición genética, este es el caso del polimorfismo de la Apolipoproteína E y su relación con el desarrollo de enfermedad aterosclerótica $(12,15)$.

El presente estudio evaluó la asociación existe entre un polimorfismo especifico de Apo E y la pre- sencia de placa aterosclerótica utilizando Reacción en Cadena de la Polimerasa y enzima de restricción Hah1 (PCR-RFLP), en una población de 122 pacientes que acudieron al servicio de cardiología de los Hospitales Santa Clara, San Blas y la Clínica San Pedro Claver en el periodo comprendido entre enero y agosto del 2005.

\section{Materiales y métodos.}

Población vulnerable y muestra: este estudio de tipo descriptivo, tomo como base una población de 2000 pacientes sin distingo de raza ni sexo con edades comprendidas entre 20 a 85 años y con posible patología cardiovascular de los Hospitales San Blas, Santa Clara y Clínica San Pedro Claver que consultaron por angina o infarto agudo del miocardio al servicio de cardiología durante el periodo comprendido entre enero a agosto del 2005, la selección de la mues- 


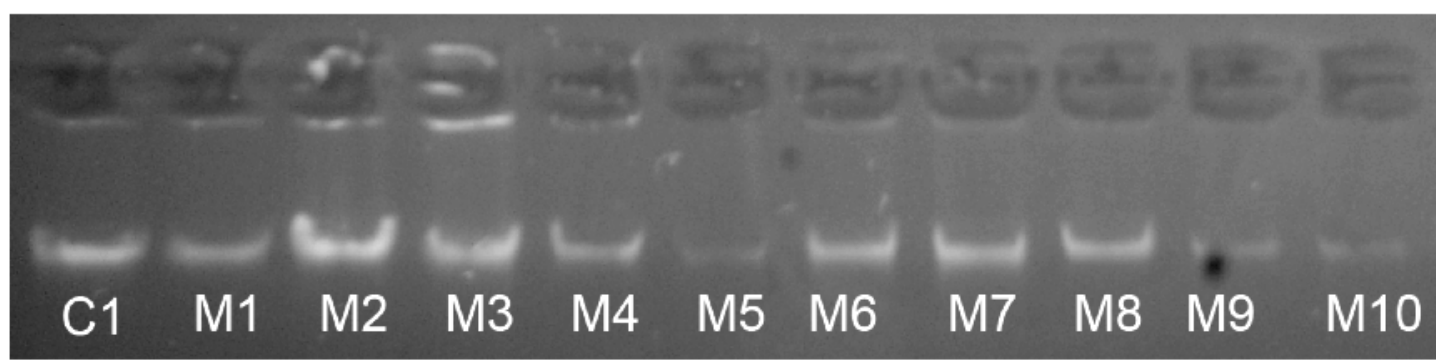

Figura 2. Chequeo obtención de DNA (2,5ul) en gel de agarosa al 1\%, 80 voltios por 50 min. C1: Patrón DNA concentración conocida (235ng/ ul). M1 a M10: Muestras extraídas con diferentes concentraciones.

tra tuvo en cuenta criterios de inclusión como: soporte de cateterismo cardiaco y coronariografia, donde se demostró o no la existencia de placas significativas; de estos, se excluyeron todos los pacientes con otros posibles factores de riesgo coronario como diabéticos, fumadores o que tomaban alcohol en cantidades mayores al consumo social. La recolección de la información se realizo por encuesta siguiendo los criterios de Helsinki al igual que la firma del consentimiento informado para participar en este estudio. Finalmente se analizaron un total de 122 pacientes, cuyas edades oscilaron entre 29 y 85 años, con peso promedio de $64.8 \mathrm{Kg}$, y talla $160.3 \mathrm{~cm}$.

Coronariografia: la determinación del grado de obstrucción se realizo por los cardiólogos hemodinamistas del Hospital Santa Clara y Clínica San Pedro Claver empleando la coronariografia por el método de sustracción digital. La placa la definieron claramente por grados de obstrucción con un coeficiente de variabilidad del 5\%. Los grados de obstrucción están directamente relacionados con la severidad de la patología coronaria generalmente, de tal manera que un grado 1 se considera arteria normal, el grado 2 leve obstrucción menor del 50\%, el grado 3 tiene compromiso de severidad medianamente alta con obstrucción del 50 al $75 \%$ y el grado 4 es de una alta severidad y compromiso obstructivo mayor del $75 \%(16)$.

Muestra de sangre: con ayuno previo de 12 horas e instrucciones de no ejercicios durante las 24 horas anteriores a la toma de muestra, se realizó una venopunción directa con aguja múltiple utilizando un tubo con anticoagulante EDTA a una concentración final de $1 \mathrm{mg} / \mathrm{ml}$ (Vacutainer).

\section{Aislamiento de DNA por el kit Wizard Genomic:}

el DNA leucocitario se obtuvo inicialmente a partir de un buffy coat de los tubos con EDTA, luego de centrifugar a $1500 \mathrm{~g} \mathrm{a} 4^{\circ} \mathrm{C}$ por $30 \mathrm{~min}$, para luego aislar finalmente el DNA utilizando un kit comercial (Promega A1120 x100) de acuerdo a las especificaciones del mismos, este método no incluye solventes como fenol y cloroformo por lo tanto los desechos no son tóxicos y son seguros para el medio ambiente. Para determinar la concentración y relativa pureza del DNA obtenido de sangre periférica, se utilizo la técnica de electroforesis con un gel de agarosa al 1\% las muestras fueron corridas al tiempo con una muestra de concentración conocida estándar usando volúmenes iguales de DNA a 80 voltios por 50 min, Figura 2.

Para verificar y validar esta concentración se realizo además una cuantificación de DNA por espectrofotometría a 260 y $280 \mathrm{~nm}$ en el Genesys 2000 utilizando 5ul de muestra para un volumen final de 100ul.

Análisis del genotipo E2, E3 y E4 de la apolipoproteina E mediante la técnica de PCR-RFLP (Polymerase Chain Reaction- Restriction Fragment Length Polymorphism): El genotipo de Apo E se determino por PCR-RFLP (Polymerase Chain ReactionRestriction Fragment Length Polymorphism) utilizando la enzima HhaI como lo describe Hixson y Vernier 


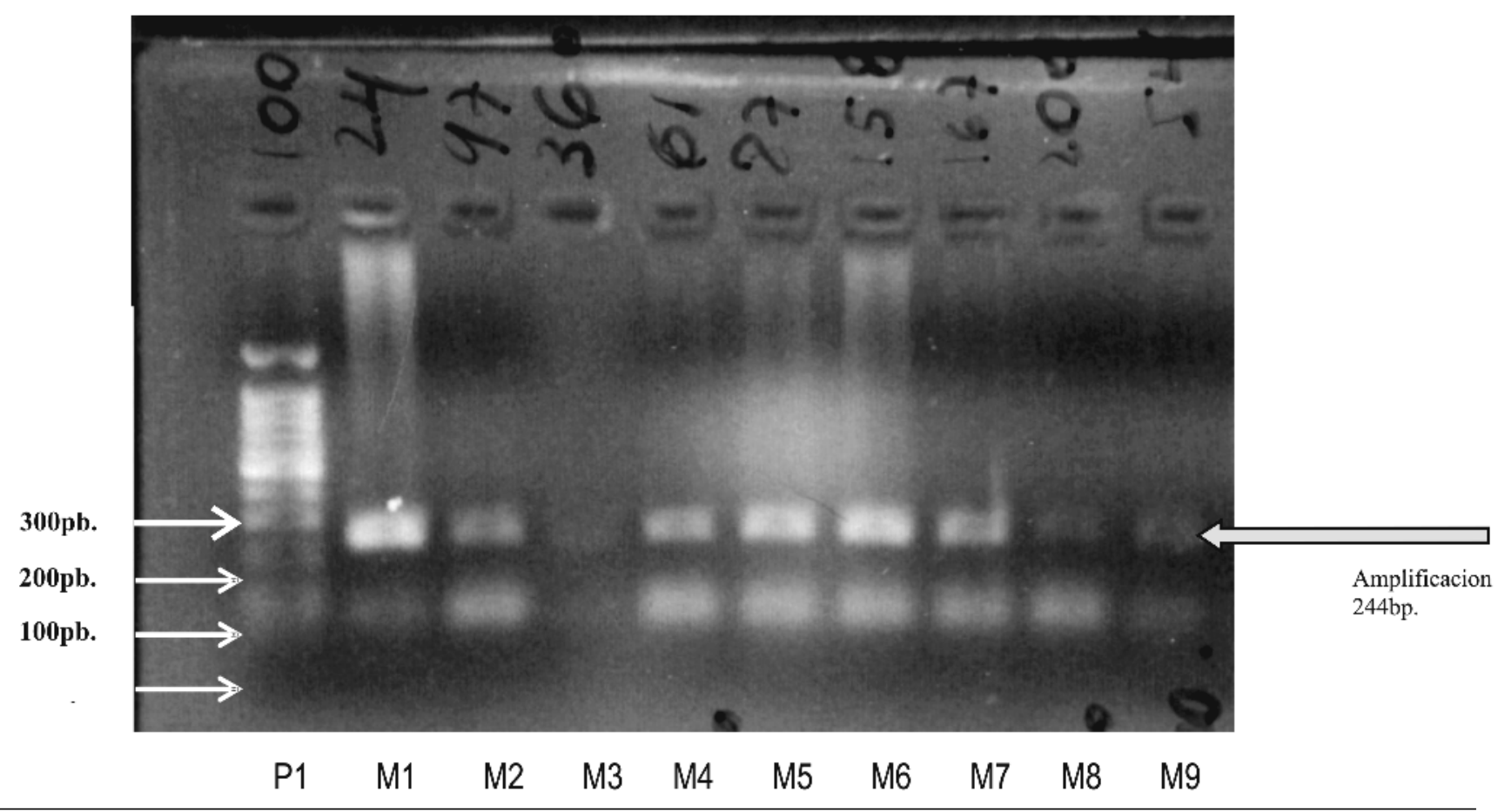

Figura 3. Chequeo amplificación de DNA (10 ul) fragmento de $244 \mathrm{pb}$ del exón 4 de la ApoE, en gel de agarosa al 1.5\% con marcador de peso molecular Promega (100bp DNA Step ladder), 80 voltios por 50 min. P1: Patrón peso molecular 100bp. M1 a M 9: Muestras amplificadas.

(17). La amplificación por PCR en el equipo My Cycler de BIO-Rad, para obtener el fragmento de $244 \mathrm{pb}$ del exón 4 del gen de la Apo E se realizo con los primers:

F4[5'-ACAGAATTCGCCCCGGCCTGGTACAC-3']y el F6 [5'-TAAGCTTGGCACGGCTGTCCAAGGA-3'] (Invitrogen life Technologies, USA), La mezcla de reacción incluyó: en un volumen final de $50 u 1$, 500ng de DNA genómico, buffer de PCR $1 \mathrm{X}(20 \mathrm{mM}$ TRis- $\mathrm{HCl}$ ph 8.4, $50 \mathrm{mM} \mathrm{KCl}$ ), $0.3 \mathrm{mM}$ de cada oligonucleótido, $0.3 \mathrm{mM}$ de una mezcla que contiene los $4 \mathrm{dNTP}, 1.5$ $\mathrm{mM}$ de $\mathrm{MgCl}_{2}, 0.5$ unidades de Taq polimerasa y dimetil sulfóxido al 10\%. La amplificación se realizó en total de 30 ciclos de el siguiente protocolo: un ciclo inicial de desnaturalización a $95^{\circ} \mathrm{C}$ por $3 \mathrm{~min}$, luego el anillamiento o alineación de matrices a $60^{\circ} \mathrm{C}$ por 30 seg y la elongación o extensión a $72^{\circ} \mathrm{C}$ por $1 \mathrm{~min}$., luego 29 ciclos consecutivos de $95^{\circ} \mathrm{C}$ por 50 seg., $60^{\circ} \mathrm{C}$ por 30 seg. y $72^{\circ} \mathrm{C}$ por $1 \mathrm{~min}$, para terminar con un ciclo de extensión de $72^{\circ} \mathrm{C}$ por $10 \mathrm{~min}$ y finalizando con ciclo indefinido de $10^{\circ} \mathrm{C}$. La confirmación de esta amplificación se realizo en gel de agarosa al $1.5 \%$ con marcador de peso molecular Promega (100bp DNA Step ladder),
Figura 3. Luego se procedió a la digestión de muestras ya amplificadas con enzima HhaI, utilizando alícuotas de $15 \mathrm{ul}$ incubadas a $37^{\circ} \mathrm{C}$ por durante 16 horas y los fragmentos obtenidos se separaron por electroforesis en un gel de poliacrilamida al $10 \%$ por 5 horas a 150 voltios hasta obtener un corrido de $9 \mathrm{~cm}$. Luego de la electroforesis los geles fueron sumergidos en bromuro de etidio a una concentración de $10 \mathrm{mg} / \mathrm{ml}$ por $15 \mathrm{~min}$. Los fragmentos de restricción se visualizaron con ayuda de un transiluminador UV y fotografiados con cámara digital adaptada para UV. Se utilizo el marcador de peso molecular de Promega (25bp DNA Step Ladder Fig 4) y controles internos de genotipos conocidos E3/ $3, \mathrm{E} 3 / 2, \mathrm{E} 4 / 4, \mathrm{E} 4 / 3$.

Análisis estadístico: Inicialmente se realizo un análisis estadístico unívariado de frecuencias para describir y conocer el comportamiento de las variables cualitativas de la población objeto de estudio, Para el análisis de asociaciones significativas entre grupos de variables, se utilizo la prueba "Chi cuadrado" para variables cualitativas categóricas y nominales como los 
grados de obstrucción de la placa aterosclerótica, genotipos de apoE codificados en polimorfismos $\mathrm{E} 3 / 3$, $\mathrm{E} 3 / 2, \mathrm{E} 4 / 4$ y E4/3 con el genero, determinando significancias estadísticas importantes .

\section{Resultados}

Se estableció que el promedio de la edad en el grupo de estudio era de 61.4 años cuyas edades oscilaron entre 29 años y 85 años; con respecto a la variable de peso se encontró un peso promedio de 64.8 $\mathrm{Kg}$, con un mínimo de $40 \mathrm{Kg}$ y un máximo de $100 \mathrm{Kg}$, la talla de estos pacientes osciló entre 139 y $187 \mathrm{~cm}$. con un promedio de $160.2 \mathrm{~cm}$, Tabla 1.

De otro lado la Tabla 2 demuestra que la obstrucción de mayor frecuencia observada en la muestra estudiada, es la del grado 3 con $48.4 \%$, en segundo lugar le sigue la no presencia de placa o grado 0 con una frecuencia de $37.7 \%$, en tercer lugar la obstrucción grado 2 muestra una frecuencia relativa de $9.8 \%$ y la menor obstrucción o grado 1 presenta la frecuencia mas baja $4.1 \%$.

Después de analizar la distribución de obstrucción de arterias coronarias estratificada por géneros, se demostró que en los hombres el porcentaje de obstrucción en grado 1, 2 o 3 es mas alto (74.6\%) con respecto a la encontrada en las mujeres (47.3\%). Esto significa que en nuestra muestra el género puede determinar un cierto grado de riesgo para presentar algún porcentaje de obstrucción importante, Tabla 3.

Es así como en los hombres con grado 3 de obstrucción, el polimorfismo predominante en un mayor porcentaje es el polimorfismo E3/3 (85\%) del total en estos sujetos $n=40$, como lo indica la Tabla 4 . El comportamiento de esta variable en las pacientes mujeres es muy similar, ya que el porcentaje mayor también lo determina el polimorfismo E3/3 (78.9\%) del total de pacientes $n=19$, Tabla 5 .

De hecho la presencia de obstrucción en los hombres con respecto a las mujeres es mayor y se analizo esta variable para encontrar algún grado de significancia estadística, encontrando que el 59.7\% del total de hombres exhiben una placa con obstrucción mayor del 70\% (grado 3), siendo el genotipo E3/ 3 el que mostró la mayor ocurrencia $(85.0 \%)$. Al analizar el "p" (0.0054) se concluye que la presencia de obstrucción en el genero masculino tiene significancia estadística importante, Tabla 6.

De otro lado en el grupo femenino del estudio $(n=55)$ el comportamiento predominante es la no obstrucción de las arterias coronarias con un porcentaje de $52.7 \%$ y el polimorfismo predominante en esta no obstrucción es el E3/3 con un 54.5\% del total de 29 pacientes analizadas. Al analizar el comportamiento de este grupo de estudio con respecto al grado de obstrucción mayor del 70\% se identifica un 34.5\% de ocurrencia y su polimorfismo predominante es el E3/ 3 con un $78.9 \%$. El "p" analizado en esta asociación (0.977) no es significante por consiguiente no hay relación entre grado de obstrucción y genero femenino ya que el comportamiento predominante en este grupo es la no obstrucción, Tabla 7.

Este comportamiento es contradictorio al ocurrido en los hombres, aunque existe similitud en el comportamiento por géneros para la presencia del genotipo E3/3, es así como en las mujeres equivale al $80.0 \%$ y en los hombres a $79.1 \%$ del total de pacientes $(n=122)$, este es independiente de la presencia o no de placa, porque, al realizar la asociación entre la ApoE 3/3 y la presencia de placa ateroesclerótica estratificada por género se encontró significancia estadística sólo en hombres; $\mathrm{X} 2=23.3$ y $\mathrm{p}<=0.005$ y X2 mujeres $=1.18$ y $\mathrm{p}=0.977$, Tablas 6 у 7 .

\section{Discusión}

El presente estudio es una de las primeras propuestas en Colombia, al menos para nuestro conocimiento actualmente, que busca asociar el grado de severidad de la obstrucción coronaria y el polimorfismo de Apolipoproteína E en pacientes coronarios para de esta manera lograr acercamientos en el conocimiento de nuevos posibles factores de riesgo coronario en busca de un factor predictivo 
Tabla 1. Distribución por promedios de edad, peso y talla de pacientes con diagnóstico de angina o infarto agudo del miocardio del servicio de cardiología de los Hospitales San Blas, Santa Clara y la Clínica San Pedro Claver de la ciudad de Bogota durante el periodo enero a agosto de 2005.

\begin{tabular}{ccccc}
\hline Variable & Media & Minimo & Máximo & DS \\
\hline Edad (años) & 61.4 & 29 & 85 & $+/-11.56$ \\
Peso $(\mathrm{Kg})$. & 64.8 & 40 & 100 & $+/-11.17$ \\
Talla (cm.) & 160.2 & 139 & 187 & $+/-9.11$ \\
\hline
\end{tabular}

$\mathrm{FR}^{\star}$ Frecuencia Relativa.

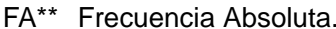

Tabla 2. Frecuencias por Grados de obstrucción de pacientes con diagnostico de angina o infarto agudo del miocardio del servicio de cardiología de los Hospitales San Blas, Santa Clara y la Clínica San Pedro Claver de la ciudad de Bogota durante el periodo enero a agosto de 2005.

\begin{tabular}{|c|c|c|c|c|}
\hline Grados de obstrucción & $\mathrm{FR}^{*}$ & $F^{* *}$ & Porcentaje Acumulado & Gráfica \\
\hline $\begin{array}{l}\text { Obstrucción }>70 \% \\
\text { Grado } 3\end{array}$ & 59 & $48,4 \%$ & $100,0 \%$ & 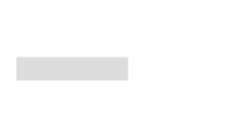 \\
\hline $\begin{array}{l}\text { No hay placa } \\
\text { Grado } 0\end{array}$ & 46 & $37,7 \%$ & $37,7 \%$ & 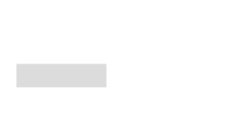 \\
\hline $\begin{array}{l}\text { Obstrucción } 50-70 \% \\
\text { Grado } 2\end{array}$ & 12 & $9,8 \%$ & $51,6 \%$ & $\square$ \\
\hline $\begin{array}{l}\text { Obstrucción < 50\% } \\
\text { Grado } 1\end{array}$ & 5 & $4,1 \%$ & $41,8 \%$ & 1 \\
\hline Total & 122 & $100,0 \%$ & $100,0 \%$ & 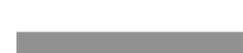 \\
\hline
\end{tabular}

$\mathrm{FR}^{*}$ Frecuencia Relativa.

FA $^{\star *}$ Frecuencia Absoluta.

Tabla 3. Distribución por tipo de obstrucción de arterias coronarias estratificada por Género de pacientes con diagnóstico de angina o infarto agudo del miocardio del servicio de cardiología de los Hospitales San Blas, Santa Clara y la Clínica San Pedro Claver de la ciudad de Bogota durante el periodo enero a agosto de 2005. $(n=122)$

\begin{tabular}{lcccc}
\cline { 2 - 5 } Obstrucción Arterias Coronarias & \multicolumn{2}{c}{ Hombres } & \multicolumn{2}{c}{ Mujeres } \\
\hline Sin Obstrucción & 17 & $25.4 \%$ & 29 & $52.7 \%$ \\
Obstrucción Grado 1, 2 y 3 & 50 & $74.6 \%$ & 26 & $47.3 \%$ \\
\hline Total & 67 & $100.0 \%$ & 55 & $100.0 \%$ \\
\hline
\end{tabular}

FR $^{*}$ Frecuencia Relativa. / FA ${ }^{* *}$ Frecuencia Absoluta. 
Tabla 4. Asociación entre polimorfismo de ApoE y obstrucción grado 3 de arterias coronarias de pacientes Hombres con diagnóstico de angina o infarto agudo del miocardio del servicio de cardiología de los Hospitales San Blas, Santa Clara y la Clínica San Pedro Claver de la ciudad de Bogota durante el periodo enero a agosto de 2005. $(n=40)$

\begin{tabular}{ccccc}
\hline ApoE Polimorfismo & FR $^{*}$ & FA $^{* *}$ & Acumulada & Gráfica \\
\hline $\begin{array}{c}\text { E } 3 / 2 \\
\%\end{array}$ & 3 & $7.5 \%$ & $7.5 \%$ & \\
E $3 / 3$ & 34 & $85.0 \%$ & $92.5 \%$ & \\
$\%$ & 1 & $2.5 \%$ & $95.0 \%$ & \\
$\begin{array}{c}\text { E } 4 / 4 \\
\%\end{array}$ & 2 & $5.0 \%$ & $100.0 \%$ \\
$\begin{array}{c}\text { E } 4 / 3 \\
\%\end{array}$ & 40 & $100.0 \%$ & $100.0 \%$ & \\
TOTAL \\
$\%$
\end{tabular}

Tabla 5. Asociación entre polimorfismo de ApoE y obstrucción grado 3 de arterias coronarias de pacientes Mujeres con diagnóstico de angina o infarto agudo del miocardio del servicio de cardiología de los Hospitales San Blas, Santa Clara y la Clínica San Pedro Claver de la ciudad de Bogota durante el periodo enero a agosto de 2005. $(n=19)$

\begin{tabular}{|c|c|c|c|c|}
\hline ApoE Polimorfismo & $\mathrm{FR}^{*}$ & $F^{* k}$ & Acumulada & Gráfica \\
\hline $\begin{array}{c}\mathrm{E} 3 / 2 \\
\%\end{array}$ & 2 & $10.5 \%$ & $10.5 \%$ & \\
\hline $\begin{array}{c}E 3 / 3 \\
\%\end{array}$ & 15 & $78.9 \%$ & $89.5 \%$ & 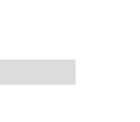 \\
\hline $\begin{array}{c}\text { E } 4 / 4 \\
\%\end{array}$ & 0 & $0.0 \%$ & $89.5 \%$ & \\
\hline$\underset{\%}{\mathrm{E}} \underset{\%}{4 / 3}$ & 2 & $10.5 \%$ & $100.0 \%$ & \\
\hline $\begin{array}{c}\text { TOTAL } \\
\%\end{array}$ & 19 & $100.0 \%$ & $100.0 \%$ & 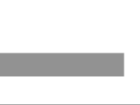 \\
\hline
\end{tabular}

molecular de enfermedad coronaria y de esta manera lograr acercamientos en el conocimiento de nuevos posibles factores de riesgo. Para este fin es indispensable retomar estudios anteriores de grupos de investigación a nivel nacional e internacional y evidenciar el grado de similitud o disparidad encontrado en los diferentes análisis, tal es el caso de BednarskaMakaruka et al 2001 (18), el cual hace parte del Proyecto WHO-MONICA y POL-MONICA con un grupo de sujetos polacos que son comparables en promedio de edad (62.0 años) y genero, $54 \%$ hombres y
$45.8 \%$ mujeres, respecto a los sujetos de nuestro estudio: edad promedio 61.4 años y $54.9 \%$ hombres con $45.1 \%$ de mujeres, presentan resultados semejantes en el alelo E3 frecuencia de $81.8 \%$ respecto a la de este estudio, alelo E3 (incluye E3/3 y E3/2) en un $89.3 \%$ (109 pacientes.)

Se puede analizar, de acuerdo a los datos reportados en este estudio que con respecto al alelo E4 (incluye E4/4 y E4/3) presentan una frecuencia de $10.65 \%$ (13 pacientes), los cuales son semejantes a los encontrados en población hispana $10 \%$ como lo 
Tabla 6. Asociación entre polimorfismo de ApoE y obstrucción de arterias coronarias de pacientes Hombres con diagnóstico de angina o infarto agudo del miocardio del servicio de cardiología de los Hospitales San Blas, Santa Clara y la Clínica San Pedro Claver de la ciudad de Bogota durante el periodo enero a agosto de 2005. $(n=67)$

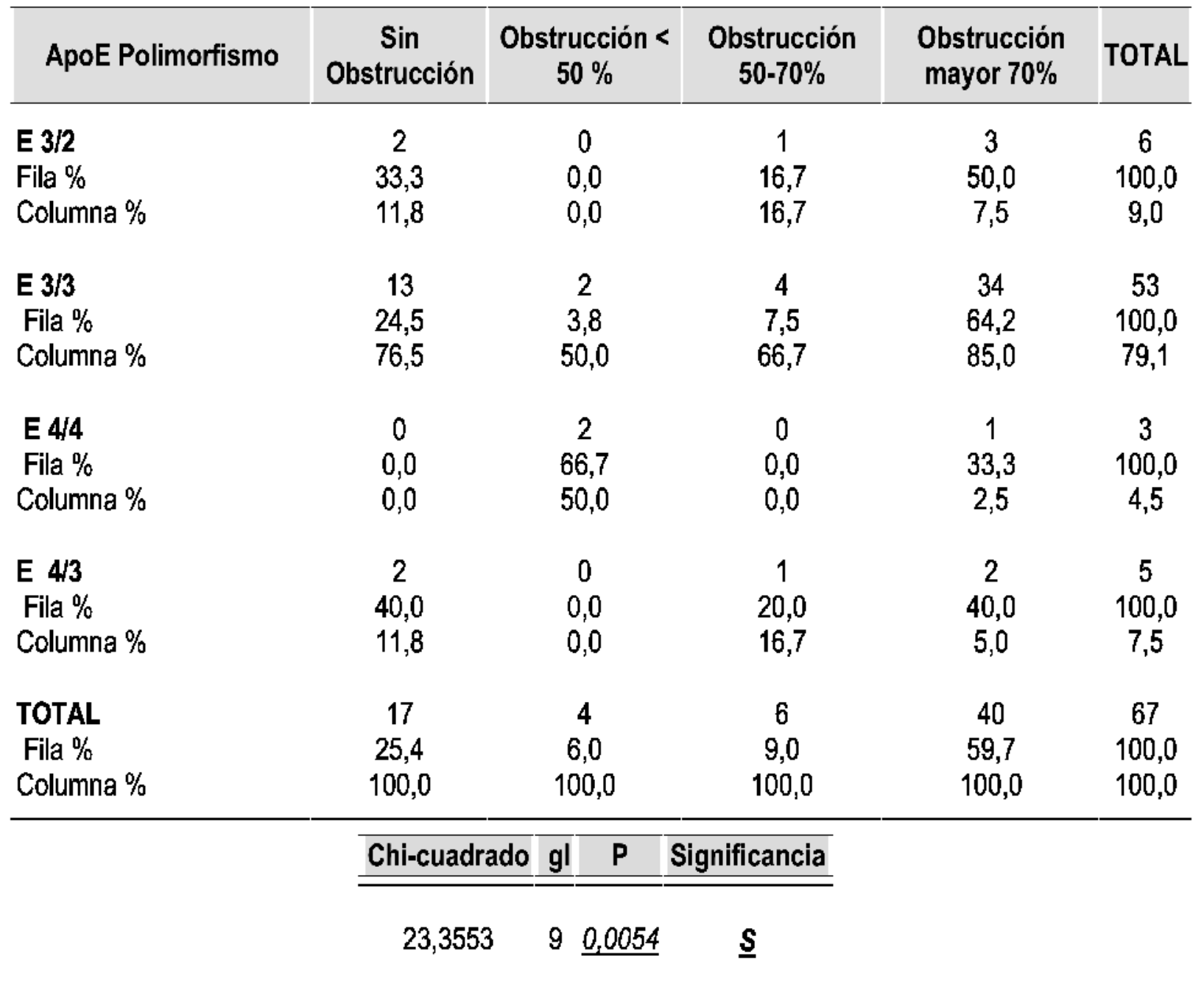

reporta García, AM. 2003 (19) en la revista Cardiológica Colombiana.

Al igual que reportan otros estudios como Davignon J and Gregg RE 1988 (20), el comportamiento del alelo E2, no se encuentra relacionado o presente en enfermedad coronaria, en el que evidencian mas que un efecto aterogénico, un efecto protector del alelo E2; otros estudios mas amplios como el Framingham Heart Study de Elosua, R. et al 2004 (21), concluyen que el alelo E2 se relaciona con bajos niveles de obstrucción. Bednarska-Makaruka et al 2001 (18) y encuentra en el alelo E2 una baja prevalencia de enfermedad coronaria. Este estudio concluye conceptos similares entorno de la no asocia- ción o presencia del alelo E2 en la enfermedad coronaria, aun sin entender realmente su efecto.

Al comparar estudios revisados en la literatura, algunos autores han encontrado asociación entre Apo E 4 y formación de placa aterosclerótica como es el caso de Stengard et al 1998 (22), Gamboa y colaboradores 1999 (19) Bennouar, N et al 2004 (23) y Elosua, R. et al 2004 (21), Kolovou et al 2002 (24); mientras que otros por el contrario no relacionan la presencia del genotipo E4 con la presencia de obstrucción y afirman que es un factor independiente como Yang, Z. et al 2001 (25).

De acuerdo con los hallazgos obtenidos en el grupo de estudio, no se encontró asociación estadística de 
Tabla 7. Asociación entre polimorfismo de ApoE y obstrucción de arterias coronarias de pacientes Mujeres con diagnostico de angina o infarto agudo del miocardio del servicio de cardiología de los Hospitales San Blas, Santa Clara y la Clínica San Pedro Claver de la ciudad de Bogota durante el periodo enero a agosto de $2005(n=55)$

\begin{tabular}{|c|c|c|c|c|c|}
\hline ApoE Polimorfismo & $\begin{array}{c}\text { Sin } \\
\text { Obstrucción }\end{array}$ & $\begin{array}{c}\text { Obstrucción < } \\
50 \%\end{array}$ & $\begin{array}{c}\text { Obstrucción } 50 \text { - } \\
70 \%\end{array}$ & $\begin{array}{c}\text { Obstrucción mayor } \\
70 \%\end{array}$ & r TOTAL \\
\hline$E 3 / 2$ & 3 & 0 & 1 & 2 & 6 \\
\hline Fila \% & 50,0 & 0,0 & 16,7 & 33,3 & 100,0 \\
\hline Columna \% & 10,3 & 0,0 & 16,7 & 10,5 & 10,9 \\
\hline$E 3 / 3$ & 24 & 1 & 4 & 15 & 44 \\
\hline Fila $\%$ & 54,5 & 2,3 & 9,1 & 34,1 & 100,0 \\
\hline Columna \% & 82,8 & 100,0 & 66,7 & 78,9 & 80,0 \\
\hline$E 4 / 4$ & 0 & 0 & 0 & 0 & 0 \\
\hline Fila \% & 0,0 & 0,0 & 0,0 & 0,0 & 100,0 \\
\hline Columna \% & 0,0 & 0,0 & 0,0 & 0,0 & 0,0 \\
\hline E $4 / 3$ & 2 & 0 & 1 & 2 & 5 \\
\hline Fila \% & 40,0 & 0,0 & 20,0 & 40,0 & 100,0 \\
\hline Columna \% & 6,9 & 0,0 & 16,7 & 10,5 & 9,1 \\
\hline TOTAL & 29 & 1 & 6 & 19 & 55 \\
\hline Fila \% & 52,7 & 1,8 & 10,9 & 34,5 & 100,0 \\
\hline Columna \% & 100,0 & 100,0 & 100,0 & 100,0 & 100,0 \\
\hline
\end{tabular}

\section{Chi-cuadrado. gl. $\quad$ P Significancia}

\section{$1,1823 \quad 6 \underline{0,9777} \quad \underline{N S}$}

Apo E 4 con la presencia de obstrucción coronaria, esto puede estar relacionado con la baja frecuencia encontrada en la población de estudio del genotipo E4 pero también podría fortalecer estudios similares que encuentran no asociación de este alelo en enfermedad coronaria, como los anteriormente mencionados o recientes como Fernández-Miranda et al 2004 (26).

Todos los grupos de investigación incluyendo nuestro estudio sugieren nuevas investigaciones y más análisis concluyentes para dilucidar mejor el efecto del alelo E4 a nivel cardiovascular.
Aunque la asociación del alelo E3/3 con respecto a la presencia de obstrucción coronaria pone a la vista en este estudio un cierto grado de significancia estadística únicamente en hombres $\mathrm{p}=0.005$, es importante definir que a diferencia algunos autores internacionales han reportado significancia y relevancia de este gen como alto riesgo de enfermedad arterial coronaria (CAD) en mujeres, como es el caso de Hirashiki A. et al 2003 (27) y Wilson, PW. et al 1994 (28). En los tres casos se concluye igualmente que la genotipificación de este polimorfismo puede dar premisas de un posible riesgo genético en CAD. 
Así como Wilson, PW. et al 1994 (28), en su estudio de Framingham Offspring que concluye que los alelos de la Apolipoproteína E son importantes marcadores genéticos de dislipidemia y CAD luego de ajustar variables de confusión al estudio, como hipertensión, obesidad, cigarrillo, diabetes, lípidos y lipoproteínas, así también nuestro estudio realizó de igual manera un acercamiento a esta deducción para dejar abiertos los espacios en busca de un conocimiento mas certero en el tema.

En particular este estudio muestra que existe significancia estadística para la presencia de obstrucción por genero en los hombres respecto a las mujeres siendo el alelo E3/3 el de mayor ocurrencia en hombres $(85 \%)$ de un total de 40 sujetos con grado 3 de oclusión lo que concuerda con estudios como el de Lehtinen et al 1995 (29) donde reporta una ocurrencia de E3/3 en un $79 \%$ en hombres aunque para este grupo de investigación no hubo diferencia significante estadística con respecto a CAD.

Al analizar los alelos ApoE 3 y Apo E 4 en conjunto con la presencia de placa aterosclerótica, no se observo asociación como lo demuestra Manssur et al 2000 (30), con el estudio de análisis de regresión multivariada en que solamente se encontró relación con otros genes distintos de apo E de tal manera que el impacto del polimorfismo como factor de riesgo en la enfermedad coronaria sigue siendo incierta. Como lo describe el metanálisis de Yiging et al en 2004 (31) donde se analizo estudios cualitativos y cuantitativos desde 1966 a la fecha; dentro de los inconvenientes que tiene este estudio es no incluir artículos que no sean en otro lengua distinta al ingles, la inadecuada escogencia de un modelo estadístico sin poder de definición de los artículos analizados, la distribución étnica y geográfica, la frecuencia alelica, la interacción del ambiente sobre el factor genético y que un gen no necesariamente significa una proteína; todo esto pueden estar contribuyendo a la no identificación de la Apolipoproteína E como factor de riesgo en enfermedad coronaria.
Dentro de las limitaciones importantes encontradas en este estudio es el hecho de ser "tipo observacional" donde las variables pueden generar confusión estadística y no ofrecer poder o peso significativo pero, se planeo así con el fin de establecer acercamientos en el tema para plantear futuros estudios con mayor valor estadístico e inferencia. También el hecho de ser observacional específico de unas Instituciones no garantiza una heterogeneidad en la muestra.

Otra limitante importante encontrada es la escasa literatura reportada en población Colombiana con estas características que se encuentre publicada en revistas indexadas, ya que en su gran mayoría los análisis realizados y artículos revisados fueron en idioma ingles y el mayor inconveniente encontrado es el hecho de carencia de homogeneidad en las poblaciones.

Finalmente, no hay resultados o estudios concluyentes entre polimorfismos ApoE y placa aterosclerótica, por lo que es indispensable plantear estudios de asociación causal a nivel del país de tipo analítico, casos y controles, y cohorte; para de esta manera poder hacer comparaciones de mayor valides externa, determinar OR con significancia entre variables y así lograr argumentar con peso estadístico el efecto genético verdadero de la Apolipoproteína E en la enfermedad coronaria obstructiva.

Estas consideraciones nos permiten únicamente plantear que este estudio es tan solo un acercamiento al comportamiento y relevancia del polimorfismo de la Apolipoproteína E en una población con obstrucción coronaria, permitiendo concluir que sigue sin ser entendido el efecto a nivel cardiovascular de la Apolipoproteína E, razón por la cual amerita mas estudios de tipo analítico específicamente y evidenciar de esta manera un comportamiento de asociación mas certero, que genere futuras investigaciones para clarificar dicho paradigma.

Es claro que en Colombia son pocos los estudios encontrados con respecto a la asociación entre Apolipoproteína E y obstrucción coronaria, y más aun 
en estos sujetos del estudio. Lo que hace más arduo el establecer comportamientos poblaciones.

En conclusión este estudio permitió identificar al alelo E3/3 como altamente asociado con la obstrucción coronaria en el genero masculino y mas aun si esta afectado por la variable edad como factor de riesgo, confirma además lo planteado por muchos autores a nivel internacional y nacional, que el alelo E2/2 pudiera tener un efecto antiaterogenico importante.

\section{Agradecimientos}

Agradecemos de manera muy especial al Grupo de Hemodinamia y Cardiología Intervensionista de la Clínica San Pedro Claver, Doctores: Fabio Hernández, Martín Suárez y Evalo Real, por su apoyo incondicional y a todos los pacientes de los hospitales: San Blas y Clínica San Pedro Claver de Bogotá D.C., que colaboraron con este estudio.

\section{Referencias}

1. Khout U, Khot M, Bajzer C, Sapp S, Ohman E, Brener S, Ellis S, Lincoff A, Topol E. 2003. Prevalence of conventional risk factors in patients with coronary heart disease. Jama 290: 898-904.

2. Kolovou G, Daskalova D, Mikhailidis DP. 2003. Apolipoprotein E polymorphism and atherosclerosis. Angiology 54: 59-71.

3. Myklebost O, Rogne S. 1988. A physical map of the apoprotein gene cluster on human chromosome 19. Hum Genet 28: 244-7.

4. Hriday K, Dast J, McPherson G, A.P. B, Sotirios K, Karathanasis J, Breslow L. 1985. Isolation, characterization and mapping to cromosome 19 of the human apolopoprotein E gene. J Biol Chem 260: 6240-7.

5. Utermann G, Langenbeck U, Beisiegel U, Weber W. 1980. Genetics of the apolipoprotein E system in man. Am J Hum Genet 32: 339-47.

6. Lai E, Riley J, Purvis I, Roses A. 1998. A 4-Mb High-density single nucleotide polymorphims-based map around human APOE. Genomics 54: 31-8.

7. Mahley RW. 1988. Apolipoprotein E: Cholesterol transport protein with expanding role in the celll biology. Science 240: 622-30.

8. Ginsberg HN. 1998. Lipoprotein physiology. Endocrinol Metab Clin North Am 27: 503-19.

9. Francke U, Brown MS, Goldstei JL. 1984. Assignament of the human gene for the low density lipoprotein receptor tochromosome 10: syntesis for a receptor, a ligando, and a genetics diseases. Proc Natl Acad Sci USA 81: 2826-30.

10. Pham T, Kodvawala A, Hui DY. 2005. The receptor binding domain of apolipoprotein $\mathrm{E}$ is responsible for its antioxidant activity. Biochemistry 44: 777-7582.

11. Wilson C, Wardell MR, Weisgraber KH, Mahley RW, Agard DA. 1991. Three-dimensional structure of the LDL receptor-binding doamin of human apolipoprotein E. Science 252: 1817-22.

12. Mahaley RW. 1983. Lipoprotein receptor and cholesterol homeostasis. Biochem Biophys 737: 197-227.
13. Lenzen HJ, Assman G, Buchwalsky R, Schulte H. 1986. Association of Apo E polymorphism, LDL, and CAD. Clin Chem 32: 778-81.

14. Staprans I, Pan X, Rapp J, Feingold K. 2005. The role of dietary oxidized cholesterol and oxidized fatty acids in the devolopment of atheroslcerosis. Mol Nutr Food Res 49: 1075-82.

15. Mahaley RW, Angelyn B. 1984. Type III hiperlipoproteinemia recent insights into the genetic defect of familial dysbetalipoproteinemia. Adv Intern Med 29: 385-411

16. Pineda M, Matiz H, Rozo R. 2002. Enfermedad coronaria. Bogotá. $757 \mathrm{pp}$.

17. Hixson J, Vierner T. 1990. Restriction isotyping of human apoliprotein $\mathrm{E}$ by gene amplification an d cleavage wtih HhaI. J Lip Res 31: 545-8.

18. Bednarska Makaruk M, Broda G, Kurjata P, Rodo M, Roszezynko M, Rymik S, Wehr S. 2001. Apolipoprotein E genotype, lipid levels and coronary heart disease in a Polish population group. Eur J Epidemiol 17: 789-92.

19. Gamboa R, Hernandéz G, Ramiro P, Zuñiga J, Granados J. 1999. Polimorfismo de la Apolipoproteina E y sus asociaciones con enfermedad cardiovascular. Arch Inst Cardiol Mex 69: 375-82.

20. Davignon J, Gregg RE, Sing CF. 1988. Apolipoprotein E polymorphism and atherosclerosis. Arteriosclerosis 8: 1-21.

21. Elosua R, Ordovas J, Cupples LA, Fox CS, Polak J, Wolf P, D'Agostino RB, O’Donnell J. 2004. Association of APOE genotype with carotid atheroslcerosis in men and women. J Lipid Res 45: 1868-75.

22. Stengård JH, Weiss KM, Sing CF. 1998. An ecological study of association between coronary heart disease mortality rates in men and the relative frequencies of common allelic variations in the gene coding for apolipoprotein E. Hum Genet 103: 234-441.

23. Bennouar N, Allami A, Laraqui A, Azeddoug H, El-Kadiri N, Benkouka F, Bendriss A, Ghannam R, Benomar A, Fellat S, Benomar M. 2004. Apolipoprotein E and angiotensinconverting enzyme gene polymorphisms as risk factors of coronary disease. Ann Biol Clin 62: 295-304.

24. Kolovou G, Yiannakouris N, Hatzivassiliou M, Malakos J, Daskalova D, Hatzigeorgiou G, Cariolou M, DV. C. 2002. Association of apolipoprotein E polymorphism with myocardial infarction in GreeK patients with coronary artery disease. Curr Med Res Opin. 18: 118-24.

25. Yang Z, Zhu T, Ma G, TYin H, Qian W, Zhang F, Cao K, Ma W. 2001. Apolipoprotein E polymorphism in the early onset of coronary heart disease. Chin Med J 114: 983-5.

26. Fernandez-Miranda C, Aranda JL, Martin MA, Arenas J, Nunez V, Gomez D1CA. 2004. Apolipoprotein E polymorphism and carotid atherosclerosis in patients with coronary disease. Int J Cardiol. 94: 209-12.

27. Hirashiki A, Yamada Y, Murase Y, Suzuki Y, Kataoka H, Morimoto Y, Tajika T, Murohara T, Yokota M. 2003. Association of gene polymorphisms with coronary artery disease in low- or high-risk subjects defined by conventional risk factors. J Am Coll Cardiol 42: 1429-37.

28. Wilson PWF, Myers RH, Larson MG. 1994. Apo E alleles, dyslipidemia, and coronary heart disease: Tha Framingham Offspring Study. Jama 272: 1666-71.

29. Lehtinen S, Lehtimäki T, Sisto T, Salenius J, Nikkila M, Jokela H, Koivula T, Ebeling F, Ehnholm C. 1995. Apolipoprotein E polymorphism, serum lipids myocardial infarction and severity of angiographically verified coronary artery disease in men and women. Atherosclerosis 114: 83-91.

30. Mansur A, Annicchino Bizzacchi J, Favaroto D, Avakian S, Cesar L, Ramires J. 2000. Angiotensin converting enzyme an $\mathrm{d}$ apolipoprotein s genes polymorphism in coronary artery disease. Clin Cardiol 23: 335-40.

31. Song Y, Stampfer MJ, Liu S. 2004. Meta-Analysis: Apolipoprotein E Genotypes and Risk for Coronary. Heart Disease 141: 137-47. 